HOUR-GLASS CONTRACTION OF THE UTERUS, AND RETAINED PLACEN'TA.

By A. J. Burmester, Esq. M.R.C.S.L., late house-surgeon at St. Bartholomew's and the Gereral Lying. in Hospitals.

EARLY in the morning, Mrs. N. N., who has always enjoyed excellent health, was taken in labour with her first child. At half-past nine A.M., the os uteri was dilated to the size of a fiveshilling piece, the membranes ruptured, and the head presenting in the first position; the bowels had been freely relieved, and the bladder emptied of its contents. The pains continued pretty regular up to about seven P.M., when they became irregular, the head at the time occupying the cavity of the pelvis; they diminished in power, and long intervals of rest were left between them; they were of that tantalizing character, that even the patient implored they might become stronger and last longer. They, however, continued in this condition until about half-past nine P.M., when, after a careful examination, finding the parts sufficiently prepared and in a fit condition, it was deemed proper to administer some ergot of rye. A scruple dose was accordingly given, and ten minutes afterwards regular pains came on, with increased vigour and in quicker succession, and terminated the birth of a fine boy, at eleven P.M. The uterus did not contract properly, but increased in size; and, on examination, it was ascertained that blood was oozing from the os externum. Cold was immediately applied to the abdomen, and pressure used in that situation, at the same time that the dose of ergot was repeated, yet no gush from the vagina followed, and the uterus remained uncontracted. Considering that the placenta was either wholly or in greater part detached, and by its presence was keeping up the hæmorrhage, the hand, guided by the cord, was introduced into the uterus, the cavity of which, as well as that of the vagina, ras filled with coagula, on the removal of which an irregular contraction of circular fibres of the uterus was detected, dividing its cavity into two compartments, in the most superior of which a portion of the placenta was found acherent. The adhesions having been broken down, it, together with as many coagula as possible, was brought away, at the same time that pressure was made on, and cold applied to, the abdomen. The uterus, notwithstanding the removal of the placenta, did not fairly contract, cold water was therefore injected into its cavity, another dose of ergot given, and the child applied to the breast, at the same time that the cold and pressure to the abdomen were continued. 'These means did at last produce contraction, and after a gush or two from the vagina, the hremorrhage ceased, leaving the patient, who had borne everything with the utmost fortitude, in a very low condition, and complaining of soreness of the throat. On placing my hand in the situation alluded to, I was surprised to feel that peculiar crackling so characteristic of the presence of air in the cellular tissue, and examining more minutely, I found emphysema occupying the throat and extending over the upper part of the thorax, evidently resulting from the patient having endeavoured to assist her pains by straining and bearing down, which should teach us to impress upon their minds the necessity of not doing so until they are unable to avoid it - namely, until the head enters the vagina and the abdominal muscles co-operate involuntarily with the contractions of the uterus.

The patient's recovery has been very rapid and favourable, so much so, that on the eighth day after her confinement she was able to leave her bed and reciine for some hours on a sofa. The emphysema has gradually subsided, without any interference, and both mother and child are in excellent health.

PRESENTAtion OF RIGHT ARM bY THE SIDE OF head.

Mrs. O'Brien, aged twenty-four, who has generally enjoyed good health, and menstruated regularly, was taken in labour, at the full time, with her second child, on the moraing of the 14th of July, 1844. On being sent for at about half-past five P.M. of the same day, I found the os uteri fully dilated and the membranes protruding, yet I was unable to make out any presentation, the parts being still high up and out of reach, from which circumstance, considering the patient had only had one child previously, I anticipated an abnormal presentation. About half an hour afterwards the membranes gave way, and on examination, the right arm was found prolapsed by the side of the head. Deeming it expedient to alter, as soon as possible, the position of the child from a faulty to a natural presentation, I proceeded to turn, and introduced my hand for that purpose, when it struck me, that by returning the prolapsed arm and keeping it up beyond the head during a pain or two, (in the same way as one would a prolapsed cord,) I might succeed in getting the head engaged in the pelvis, and so prevent the arm from again descending, and thus render the operation of turning unnecessary. This was accomplished after two pains, when the head entered the pelvis in the first cranial position, and labour was terminated, by the natural powers, at half-past ten P.M., when a healthy boy was born with the cord twice round his neck. About ten minutes afterwards the placenta was found entirely detached in the vagina, and brought away, the uterus being firmly contracted.

This case proves the value, and points out the necessity, of an early and careful examination, for if the prolapsed arm had not been detected in time, and this case had been left to nature, I am convinced a cross-birth would have been the result, the head resting in the left and the pelvis of the child in the right iliac fossa of the mother, and the severe operation of turning would have been rendered indispensable.

Starhope-street, Gloster Gate, Regent's Park, Dec. 1844.

\section{ON THE USE OF THE TOBACCO ENEMA IN HERNIA.}

By W. Chuley, Esq. Surgeon, Ashton-under-Lyne.

I woULd beg a corner in THE LANCET for a remark or two on the use of opium and tobacco, in cases of hernia; the former remedy having failed, in such opportunities as I have had, to produce the effects which might be expected from the accounts of late writers, and the objection against tobacco from its dangerous character being obviated by the use of means, which, although only tried in one case, yet in that with such marked effect as warrants the expectation that a similar result will generally follow. When op:um fails, which I believe it frequently will, the relief it affords from present suffering is no small objection to its use, inasmuch as the false idea of security which such relief is calculated to inspire may lead to the dangerous delay of more efficient measures. The plan, then, that I refer to, as obviating the dangerous effects of tobacco is, simply, the removal of the infusion from the bowels after it has produced its full effect.

Octuber 27, 1844, half-past ten A.M. Was called to Mrs. B., aged 48 , and mother of sixteen children. Found her suffering from femoral hernia, which had been in a state of strangulation all the morning. The hernia had existed four months, and had never once been returned. I tried the taxis and cold applications ineffectually, then administered a tobacco enema of the usual strength, and left her for about an hour. I returned, accompanied by Mr. Booth, of this town, and found her in a most alarming state of prostration. The tumour was less tense, and, after repeated manipulations, at last returned under the hands of $\mathbf{M r}$. Booth, but the occurrence of death seemed not the less likely to happen.

The plan I have alluded to was now put into operation. Water was injected as long as it would pass, and allowed to return by the tube, for the bowels seemed to have lost all expulsive power. This was repeated three or four times before signs of reaction took place, which was, however, soon satisfactorily established; and at a visit some two hours afterwards, the safety and gratitude of the patient were equally apparent.

Ashton-under-Lyne, December, 1844.

ON PARTIAL INVERSION OF THE UTERUS AFTER LABOUR.

By Jonam Clarkson, Esq. late Resident Surgeon of the Birmingham Lying-in Hospital.

I wAs called, at half-past ten o'clock, on the evening of Thursday, the 7th of March, 1844, to attend Mrs. Cthirty-four, a short, stout woman, then in labour of her eleventh child. On my arrival at her residence, about a quarter of an hour afterwards, I found her walking about the house, apparently very comfortable, having had but few pains, and those at long intervals, during the day. She informed me that all her other labours had been quick; and as the membranes had. ruptured at three o'clock in the afternoon, nearly eight hours before my visit, she felt convinced that her labour would speedily be terminated. As she walked across the room, I observed her abdomen to be unusually prominent, and suspected she might have twins; but in this I was mistaken. At my request she placed herself on the bed, so that an examination per vaginam might be made, when I discovered the head already low down in the cavity of the pelvis; the os uteri receded beyond the reach of the finger, and the labia and perineum soft and dilatable. As yet there had been no uterine contraction since $\mathbf{I}$ entered the room; but just as she was getting off the bed, in orjer that it might be more comfortably prepared, a violent pain came on; and almost before I could apply my hand to the perineum, the child was expelled, and the placenta brought to the os 
externum by the continuance of the same pain. Having hastily tied and divided the funis, and removed the placenta, (which was perfectly loose,) I passed my finger into the vagina to ascertain the condition of the os uteri, which I could feel high up, widely dilated, and embracing a soft, globular substance, which protruded through it, and occupied the vagina. This I at first imagined was the bag of membranes belonging to the other foetus I had suspected to be there. To satisfy myself, I placed my other hand on the abdomen, but, to my surprise, could feel nothing like the uterus there, although I made deep pressure for it. I therefore instituted a more careful examination of the tumour itself, when the following circumstances assisted me in forming a diagnosis. First. It was exceedingly sensitive; the least movement of my finger across it occasioned her great pain. Second. Hæmorrhage commenced, though not to any alarming extent. Third. Symptoms of prostration suddenly manifested themselves, more than I should have expected, either from the sudden delivery, or from the bleeding. I accordingly concluded that the present must be a case of partial inversion of the uterus, the fundus having followed, by its sudden and energetic contraction, the placenta, through the os ateri, whilst this latter remained dilated.

Acting under this conclusion, I pressed the knucleles of two fingers against the most dependent part of the tumour, and was delighted to feel it gradually giving way, until it suddenly started back into its place in the pelvis, leaving my hand occupying its cavity. I could now distinctly feel it through the abdominal parietes, firm and well contracted, although the os uteri still remained considerably dilated. The hæmorrhage immediately ceased when the inversion was reduced. After waiting an hour with her, during which time there was no symptom indicating danger, and having applied a bandage firmly round the lower part of the abdomen, I left her. Mrs. C__ had a speedy and most favourable recovery.

There are a few circumstances connected with the foregoing history that are perhaps worthy of being considered more in detail. First. This was evidently a case of spontaneous inversion, produced by the vigorous but irregular contraction of the uterus. It is undoubtedly true, that by far the greater proportion of these cases, when they do occur, arise from unskilful management in the last stage of labour, unjustifiable traction being made at the funis, for the purpose of speedily removing the placenta; whilst the uterus itself remains dilated and flaccid, so that the fundus is literally dragged down through the os internum into the vagina, where it is but too often allowed to remain, until all possibility of returning it is for ever lost. Still I think it may be fairly questioned whether traction is always the exciting cause of this accident, whether made by the hand of the accoucheur, or by the unusual shortness of the cord pulling down the placenta and fundus uteri, where it has occurred in cases of very rapid delivery : at any rate, neither of these causes could operate in the case I have related; for, in the first place, I had no opportunity of removing the placenta from the uterus, and, in the second, the funis was longer thau ordinary-a point concerning which I fully satisfied myself before leaving the room.

Every experienced accoucheur is aware that irregularities do frequently occur in the contraction of the uterus, one part becoming hard and firm, whilst another remains soft and dilated; in fact, it is from this very circumstance that so much difficulty is occasionally experienced in removing a retained placenta. I have frequently observed, especially in thin women, in whom the uterus could be distinctly felt through the abdominal walls, that, during its contractions, after the expulsion of the child, there has been for a short period a very manifest depression in one situition, but which, immediately afterwards, I have been anable to find. This circumstance, I imagine, depends upon some irregularity or inequality in the action of the uterine fibres; and the same cause acting more powerfully, particularly at the fundus of the uterus, may undoubtedly give rise to inversion. Nor is this mere speculation, for accidents of this kind now and then happen, which cannot be satisfactorily explained upon any other hypothesis; as, for example, in those cases where the child has been expelled after the death of the mother, and where the process has not been in the slightest degree interfered with.* Again, cases of inversion are recorded where, for several hours and even days after delivery, no symptom has manifested itself which could lead even the most anxious attendant to suspect the occurrence of such a fearful accident. A case is quoted in the 51st vol. of The London Medical and Physical Journal, of a woman who was rapidly recovering from a favourable labour, but who, on the tenth day from her delivery, "whilst making

- See cases referred to in the Edinburgh Medical and Surgical Journal for January, 1840 . violent efforts to evacuate the contents of the rectum, felt a bulk mass descend through the vagina, which was followed by great pain in the belly and the displaced parts, and in the groin, with strong efforts to vomit, and a sensation of faintness." The uterus was discovered to be completely inverted, and the accident had undoubtedly occurred when the straining efforts were made One of the most intelligent practitioners in Birmingham, who has deservedly enjoyed a large midwifery practice, has informed me that he once attended a woman in her labour, who was favourably recovering, having had no bad symptom, when, on the second day from her confinement, the uterus was inverted during the operation of a quantity of castor oil, which she had taken without his lsnowledge.

Other cases might be adduced of the same character, and the conclusion that forces itself upon the nind is, that inversion does sometimes take place unconnected with any traction made on the fundus uteri, either by an unusually short funis, or by the hand of the accoucheur.

Second. Of the symptoms present in the above case, two were most prominent-viz., the highly sensitive tumour occupying the upper part of the vagina, and the sudden depression of the patient. When these symptoms appear after a labour, there can be little doubt as to the nature of the accident, and the necessity for immediate and judicious treatment. I find, upon reference to the published cases of this kind, that in almost every instance, mention is made of the sudden prostration of the vital powers, as indicated by a tendency to syncope, hiccup, \&c. This, I believe, does not arise so much from the loss of blood, as from the shock produced by the sudden displacement of the parts; it is, in fact, similar to the prostration consequent upon injury inflicted upon any other organ largely supplied with the nerves of organic life.

It is not necessary to make any remark upon the first-mentioned symptom, since the discovery of a highly sensitive tumour in such a situation, and at such a time, cannot be mistaken : but I may perhaps express an opinion, that it is incumbent on every one who makes himself responsible for a woman's safety during her labour, not to leave the room until he bas carefully ascertained the condition of the os uteri, and also the situation and degree of contraction of the uterus itself, the former by an examination per vaginam, and the latter through the abdominal ralls.

Third. In no case is the importance of forming a correct diarnosis more forcibly exhibited than in one of this nature. Discovered at the time of its occurrence, nothing is more simple than its reduction, nothing more certain than its successful issue. But let the nature of the case be overlooked or misunderstood in the first instance, and it is almost equally certain that the discovery will be made too late to sare the woman from an untimely and miserable end.

Paradise-street, Birmingham, Sept. 30, 1814

\section{ON THE TREATMENT OF INDOLENT AND} IRRITABLE ULCERS.

By J. Proprck, M.D., late Physician to the St. Giles's Dispensary, London.

INTRACTABLE cases of ulcers on the legs are of frequent occurrence in our hospitals and at our dispensaries. The object of this paper is, to state the mode of treatment which I have found eminently successful in such cases, many of which came under my care during the sixteen years $I$ had the sole charge of the dispensary in St. Giles's. Many of the patients were occupied in selling fish or fruit in the streets; they were standing frequently in the wet, exposed to the weather, from early in the morning till late at night, and the women were employed in washing; their diet was chiefly fish, and that not particularly fresh; ontside rashers of bacon, decayed cheese, and rancid butter; their beverage was tea, porier, and gin; a combination of articles of food highly conducive to impurities of the blood, especially when favoured by a total neglect of personal cleanliness, and by the foul air of their dwellings at night. Under these circumstances, I regarded the nicer on the leg as a natural issue or outlet for the escape of certain morbid principles from the blood, the retention or suppression of which would have occasioned diseases of a more dangerous tendency. Hence, $I$ considered that the popular prejudice against drying up of old ulcers was not devoid of reason.

The analogy between those natural issues in the leg and artificial issues in the arm in affording relief to the system, in cases of disease from impurity of the blood, is very striking.

If the ulcer or the issue emit a disgusting odour, and discharge freely, the necessity for such a drain is unequirocal; it cannot be 